\title{
COMPUTATIONAL MODELLING OF THE RAIL VEHICLE MULTIBODY SYSTEM INCLUDING FLEXIBLE BODIES
}

Tools for computer simulations are widely used in the field of a rail vehicle design. These means of virtual reality allow to perform static analyses of rail vehicle parts and dynamic analyses of a rail vehicle multibody system. For static analyses the finite element methods are most commonly used while the multibody system analyses are used for analyses of the rail vehicle dynamics. This paper deals with an implementation of flexible bodies into the rail vehicle multibody system. The implementation of flexible bodies into the rail vehicle multibody system extends the using of simulations, especially for an assessment of an influence of flexible bodies on running properties of the rail vehicle.

Keywords: Computational modelling, rail vehicle, multibody system, flexible bodies.

\section{Introduction}

In modern development process of rail vehicle computer aided simulations are employed. In this way costly experiments and prototypes can be reduced. Production of a rail vehicle is composed of several phases. There is a design phase, a development and optimisation phase, production of a rail vehicle, verification and validation of a rail vehicle and, in the end, commissioning of a rail vehicle. At this time computer software allows to perform complex simulations. Thus, shorter development periods and rising requirements like durability, efficiency or mass reduction demand precise simulations, which intensify the usage of lightweight structures.

\section{Principles of rail vehicle multibody system with a flexible body}

The need for more accurate models of a rail vehicle to describe the complex behaviour of flexible systems experiencing large motion while undergoing small elastic deformations motivated the development of many powerful analysis techniques. The most popular formulations use time-variant mass matrices to describe the inertia coupling between the rigid body motion and the elastic deformation [1].

To describe the dynamic behaviour of a rail vehicle mechanical system which undergoes large nonlinear working motions the multibody system (MBS) approach is often most useful [2]. A classic MBS of a rail vehicle consists of rigid elements which are connected by ideal joints, coupling elements [3], contact elements [4 and 5] and force elements. The phenomena of the wheel/rail contact [6] significantly influence the rail vehicle properties and wheel/rail contact stress evaluation [7 and 8].

For applications in the field of rail vehicle analysis where the deformation of the bodies cannot be neglected, the method of flexible multibody systems has to be applied. In the flexible multibody system of a rail vehicle the approach is extended by flexible bodies.

The application of flexible bodies into the rail vehicle multibody system with the help of the finite element method introduces a large number of flexible degrees of freedom into the rail vehicle model. The reduction of the linear flexible degrees of freedom is the principal step for an efficient simulation of a flexible multibody system of a rail vehicle [9].

\subsection{Foundations of flexible multibody dynamics}

For the kinematic description of the motion of flexible bodies that are subjected to large displacements several methods are used. Among these methods are, for example, the floating frame of reference, convected coordinate system, finite segment method and large rotation vector. Large deformation problems in flexible

\footnotetext{
* ${ }^{1}$ Jan Dizo, ${ }^{1}$ Miroslav Blatnicky, ${ }^{2}$ Blanka Skocilasova

${ }^{1}$ Department of Transport and Handling Machines, University of Zilina, Slovakia

${ }^{2}$ Department of Machines and Mechanics, J. E. Purkyne University in Usti nad Labem, Czech Republic

E-mail: miroslav.blatnicky@fstroj.uniza.sk
} 
multibody system can be efficiently solved using the absolute nodal coordinate formulation [10].

In the absolute nodal coordinate formulation, neither infinitesimal nor finite rotations are used as the element coordinates. The locations and deformations of the material points on the finite element are defined in the global coordinate system using the element shape function and nodal coordinates (Fig. 1).

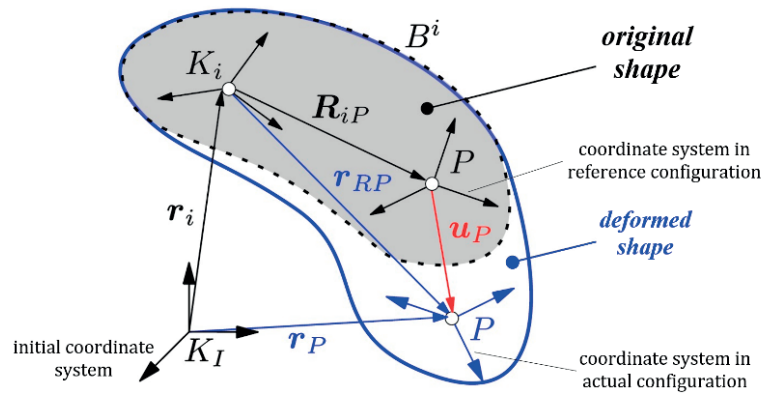

Fig. 1 Representation of the flexible body kinematics

The floating frame of reference formulation is currently the most widely used method in the computer simulation of flexible multibody systems [10 and 11]. It is used for the systems where the elastic deformation is small compared to the rigid body motion. The basic idea is to separate the motion of the body into a large nonlinear motion of the reference frame and a small linear elastic deformation with respect to the reference frame. The motion of a particular point $P$ on the flexible body $B^{i}$ is represented by the vector $r_{p}(t)$ (Fig. 1). A flexible body can be e.g. the bogie frame, the body of wagon or rail etc. Using the floating frame of reference formulation, the position vector $r_{p}(t)$ of the point is separated into a usually non-linear motion $r_{i}(t)$ of the reference frame and a superposed linear elastic deformation $u_{p}(t)=u\left(R_{i P}, t\right)$ with respect to the reference frame [11]

$r_{p}(t)=r_{i}(t)+R_{i P}+u_{p}(t)$

see Fig. 1. The vector $R_{i P}$ corresponds to the position of the point $P$ in the undeformed state.

A similar approach concerning the orientation of the coordinate system at point $P$ leads to the small rotational motion $\vartheta_{P}(t)=\vartheta_{P}\left(R_{i P}, t\right)$ by elastic deformation. Once deformation disappeared, this kinematic formulation leads to exact modelling the rigid body dynamics of a rail vehicle. When this formulation is used, the modelling of a flexible multibody system of a rail vehicle can be divided into two parts (Fig. 2) [11].

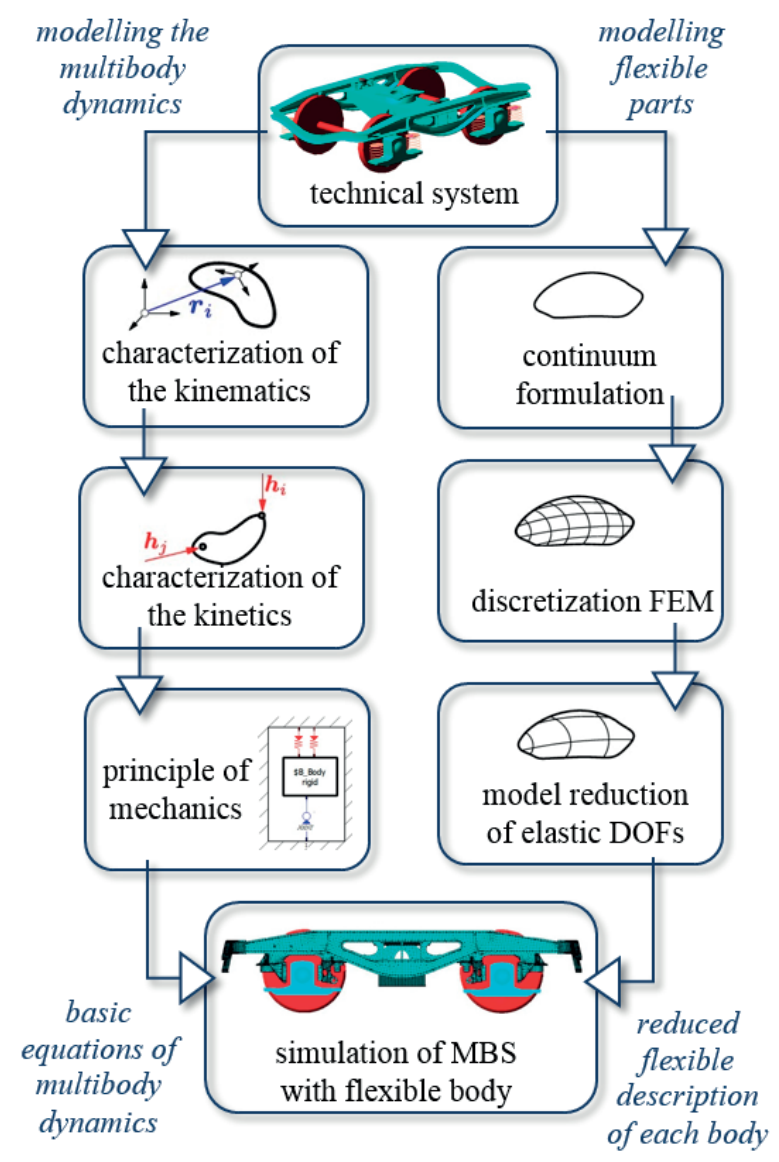

Fig. 2 Procedure of the rail vehicle multibody modelling and simulation with flexible bodies

This describes on the one hand the modelling of multibody dynamics and on the other hand the modelling of flexibility of selected bodies. Combination of these ways establishes the flexible multibody system.

\section{Modelling of the multibody system of rail vehicle with flexible bodies}

The Finite Element Method (FEM) is one of the most commonly used tools for the flexibility description [12 and 13]. It is an approximation and discretisation method for field problems formulated for differential equations. In structural dynamics the displacement field of a continuum is approximated by the Ritz approach and d'Alambert's principle to get the equation of motion of a finite element model. The linear elastic deformation $u(R, t)$ and $\vartheta_{p}(R, t)$ are specified by the Ritz approach [11]

$u(R, t)=\Phi(R) \cdot q_{e}(t), \vartheta(R, t)=\Psi(R) \cdot q_{e}(t)$, 
where $q_{e}(t)$ refers to the nodal displacements of a finite element model and $\Phi(R)$ and $\Psi(R)$ are the elastic shape functions [9]. This results in the equation of motion of a flexible body

$$
M_{e} \cdot \ddot{q}_{e}(t)+K_{e} \cdot q_{e}(t)=h_{e},
$$

as formulated in [9]. The matrices $M_{e}$ and $K_{e}$ are the mass and stiffness matrices of the flexible structure and have the following characteristics if the system is constrained sufficiently to avoid rigid body motion

$M_{e}=M_{e}^{T}>0, K_{e}^{T}>0$,

The generalised surface and volume forces are summarised in the force vector $h_{e}$. To consider the dissipative effects an additional damping matrix $D_{e}$ is often introduced and can be approximated, e.g. by viscous damping (Rayleigh damping):

$D_{e}=\alpha M_{e}+\beta K_{e}$,

with the proportional factors $0 \leq \alpha, \beta \in \mathbb{R}$. The need for high precision and complex geometries often leads to a fine spatial discretisation. Mathematically the flexible bodies are described by a large set of linear ordinary differential equations, whose solution increases the computational effort of the simulation. Linear model reduction is a decisive component to efficient simulation. To get a representation used for some model reduction techniques the forces acting on the finite element structure are described by the time dependent excitation $u_{e}(t)$ and the input or control matrix $B_{e} \in \mathbb{R}^{N \times p}$. This matrix captures the spatial distribution of the boundary and coupling conditions. Further on, the output or observation matrix $C_{e} \in \mathbb{R}^{r \times N}$ is introduced for the calculation of the interesting displacements $y(t)$. In this case, equations of motion of a single flexible body can be formulated as a linear timeinvariant second order multi input multi output system:

$$
\begin{aligned}
& M_{e} \cdot \ddot{q}_{e}(t)+D_{e} \cdot \dot{q}_{e}(t)+K_{e} \cdot q_{e}(t)=B_{e} \cdot u_{e}(t), \\
& y(t)=C_{e} \cdot q_{e}(t) .
\end{aligned}
$$

Due to increasing demands on the characteristics of technical products and their simulation, the requirements on the calculation accuracy and the calculation time are often extremely high. On the bottom line this trend means that the dimension of the equation of motion rises and the time to run the simulation should be as quick as possible. Such problems particularly require an adequate model order reduction to decrease the number of equations and keep the significant characteristics of the system. Using the floating frame of reference formulation and the linear model order reduction via projection of equation of motion (3), (5), (6) (see [9 and 11]) can be used. Therefore, the large number of degrees of freedom of the flexible coordinates $q_{e} \in \mathbb{R}^{N \times 1}$ are approximated in a subspace $\mathcal{V}_{s}$ of smaller dimension $n<N$ by the reduced displacement vector $\tilde{q}_{e} \in \mathbb{R}^{n \times 1}$

$q_{e} \approx V \cdot \tilde{q}_{e}$

This subspace $\mathcal{V}_{s}$ is described by the projection matrix $V \in \mathbb{R}^{N \times n}$. The use of this relation in FEM equations of motion (5) and (6) leads to an over-determined system and leaves a residuum because the exact solution $q_{e}$ is generally not an element of the subspace $\mathcal{V}_{s}$. To obtain a unique solution the residual should be orthogonal on a second subspace $\mathcal{W}_{s}$ represented by $W \in \mathbb{R}^{N \times n}$. The orthogonality conditions or Petro-Galerkin conditions result in the reduced $\mathrm{FE}$ equations

$\tilde{M}_{e} \cdot \ddot{\tilde{q}}_{e}+\tilde{D}_{e} \cdot \dot{\tilde{q}}_{e}+\tilde{K}_{e} \cdot \tilde{q}_{e}=\tilde{B}_{e} \cdot u_{e}$

with the reduced matrices $\tilde{M}_{e}:=W^{T} \cdot M_{e} \cdot V$, $\tilde{D}_{e}:=W^{T} \cdot M_{e} \cdot V, \tilde{K}_{e}:=W^{T} \cdot K_{e} \cdot V \in \mathbb{R}^{n \times n}$ and $\tilde{B}_{e}:=W^{T} \cdot B_{e} \in \mathbb{R}^{n \times p}, \tilde{C}_{e}:=C_{e} \cdot V \in \mathbb{R}^{r \times n}$.

The projection is called orthogonal if the subspaces are identical $V=W$ and oblique otherwise. This procedure leads to the reduced equations of motions of one flexible body:

$\left[\begin{array}{cc}M_{r}^{i} & M_{e r}^{i} \cdot V \\ W^{T} M_{e r}^{i} & \tilde{M}_{e}^{i}\end{array}\right] \cdot\left[\begin{array}{c}\ddot{q}_{r}^{i} \\ \tilde{\tilde{q}}_{e}^{i}\end{array}\right]+\left[\begin{array}{c}0 \\ \tilde{K}_{e} \cdot \tilde{q}_{e}+\tilde{D}_{e} \cdot \dot{\tilde{q}}_{e}\end{array}\right]=\left[\begin{array}{c}h_{r}^{i} \\ W^{T} \cdot h_{e}^{i}\end{array}\right]$.

A task of different reduction techniques is to find the projection matrices $V$ and $W$.

\section{Approach for the reduction of rail vehicle parts}

For the simulation of the rail vehicle multibody system with flexible bodies some preprocessing steps for obtaining a reduced flexible body are necessary. It is possible to make in FEM software, for example in Ansys [14 and 15]. Ansys software allows engineers to construct computer models or structures, machine components or system, apply operating loads and other design criteria and study physical responses [16 and 17]. This software also allows to reduce flexible bodies for import into MBS software.

The general process to integrate flexible bodies into the rail vehicle multibody system consists of several operations:

- $\quad$ setting up the finite element model,

- integrating the finite element model into the MBS software,

- setting up the MBS model of the rail vehicle.

It is needed to reduce the size (number of freedom) of FEM model before working with the MBS interface. For this it is needed to perform several operations:

- define the interface nodes. The MBS interacts with the FEM superelement on these nodes.

- connect the interface nodes with structure. In Ansys it is recommended to use the following elements types: 
- rigid body element - interface nodes have independent DOFs, coupling nodes on the FE structure have dependent degrees of freedom, dependent nodes perform rigid body motion only and independent node (interface node) defines this rigid body motion. Element types of rigid body elements for Ansys FEM code are CE, CERIG, MPC184 and RBE 2.

- force distributing constraints - interface node has dependent DOFs, coupling nodes on the FE structure have independent DOFs, motion at the interface node is the weighted average of the motion of the coupling nodes, forces and moments at the reference node are distributed either as forces or moments at the coupling nodes. Element types of force distributing constraints for Ansys FEM code are TARGE 170+CONTA173, TARGE 170+CONTA174

- define the coupling nodes as retained nodes,

- define the retained DOFs. This step is important in the reduction process for yielding accurate superelement matrices [18].
Once the FEM model of a part of the rail vehicle is reduced, the input files generation for MBS software is required. The file with the flexible body input data is necessary for including flexible bodies in the MBS software. After loading the file with the FEM input data into the MBS software it is possible to define the interaction between flexible body and MBS system by using joints, constraints or force elements which apply loads to the flexible body. The flexible body deformation is caused by these loads [15].

For the needs of a rail vehicle simulation the FEM model of a bogie frame of a freight wagon was created. This is the most commonly used bogie in the Central and Eastern Europe - the Y25 bogie [19, 20 and 21].

\subsection{Reduction of the bogie frame}

In this section the procedure of preparation of the flexible model of the bogie frame is described.

The CAD model of the bogie frame was imported into the FEM software. For the preparation of the FEM data the software

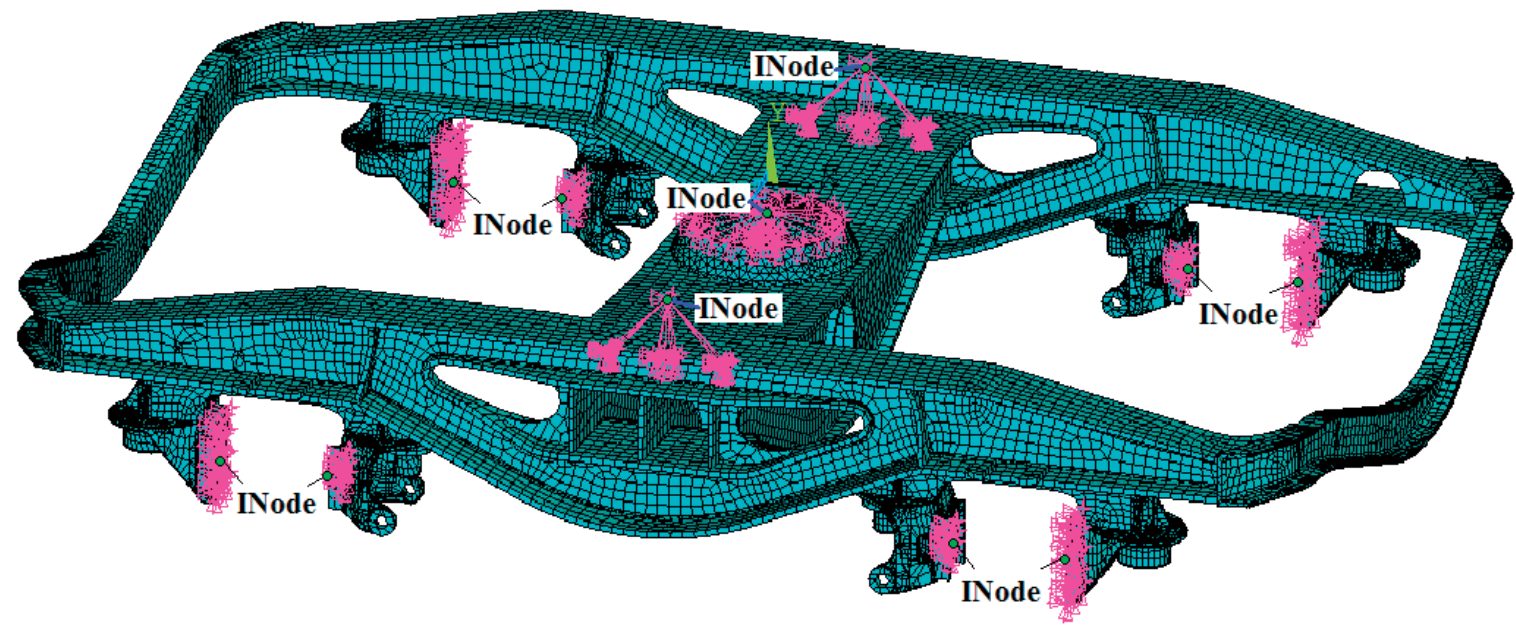

Fig. 3 FEM model of the bogie frame with interface nodes (INode)

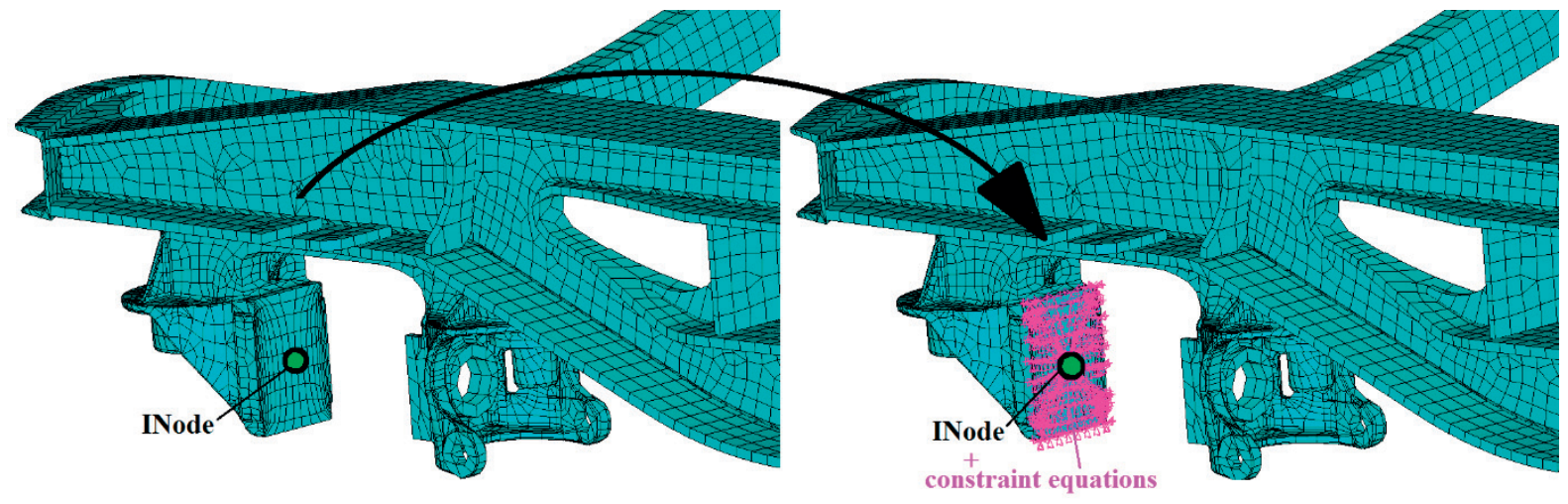

Fig. 4 Interface node (INode) on the friction surface (left) and constraint equations (right) 
Ansys was used. In Ansys the FEM mesh and simulation of flexible body behaviour were performed [14 and 22]. For interface nodes the rigid body element was used. Interface nodes were defined in locations of joints, constraints and force elements (Fig. 3). In Fig. 3 interface nodes on the bogie frame created by using rigid elements are shown.

In Fig. 4 the example of interface node (INode) and relevant constraint equations created on the friction surface of the axle guide are shown. In this interface node friction forces between the bogie frame and the axlebox in the multibody system of the freight wagon are defined.

In the future research this FEM model of a bogie frame of a freight wagon will be used for importing into the MBS model of a freight wagon. After creation of the rail vehicle multibody system with flexible bodies analyses will be performed. These analyses will allow the better assessment of the freight wagon ride properties. It will be needed to consider that the freight wagon most commonly consists of two bogies. The created FEM model of a bogie will require much bigger computer capacity and will extend the computational time significantly.

\section{Conclusion}

The computer simulation is nowadays an integral part of the development process of rail vehicles. The flexible multibody dynamics is the field that encompasses several subjects such as rigid body dynamics, continuum mechanics, finite element method, numerical and computer method. Multibody simulations with flexible bodies enable more detailed analyses of a rail vehicle behaviour. The inclusion of the flexible body into MBS simulation allows better optimization of rail vehicles design as well as prevention of potential problems during their long-term operation.

\section{Acknowledgements}

This paper was created during the processing of the project No. APVV-0842-11: "Equivalent railway operation load simulator on the roller rig". The work is also supported by the Scientific Grant Agency of the Ministry of Education of the Slovak Republic and the Slovak Academy of Sciences in project No. 1/0347/12: "Railway wheel tread profile wear research under the rail vehicle in operation conditions simulation on the test bench", project No. 1/0383/12: "The rail vehicle running properties research with the help of a computer simulation" and No. 1/1098/11: "Stress Distribution in a Braked Railway Wheel".

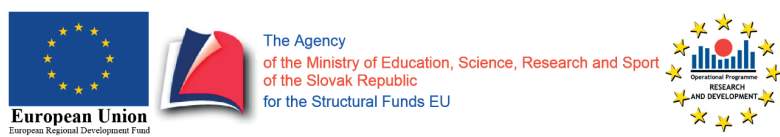

This contribution is the result of the project implementation: "Development of two types of freight wagons with bogies for non-standard wheelbase or track wheelset, complying with the criteria for interoperability, Environmental Issues, safety and reliability“, ITMS code 26220220070, supported by the Operational Programme Research and Development.

"We support research activities in Slovakia / Project is co-financed from EU sources."

\section{References}

[1] SCHIEHLEN, W.: Research Trends in Multibody System Dynamics. Multibody System Dynamics, 18, 2007, 3-13, Springer Science + Business Media B.V 2007. DOI 10.1007/s11044-007-9064-4.

[2] SAGA, M., ZMINDAK, M, DEKYS, V., SAPIETOVA, A, SEGLA, S.: Selected Methods of Analysis and Synthesis of Mechanical Systems, VTS: University of Zilina, 2009, 360 p., ISBN 978-80-89276-17-2.

[3] SAPIETOVA, A., SAGA, M., SHIMANOVSKY, A., SAPIETA, M.: Mobility of Multibody System in Terms of their Incorrectness. Communications - Scientific Letters of the University of Zilina, vol. 16. No. 3a, 2014, 33-39. ISSN 1335-4205.

[4] LACK, T, GERLICI, J.: Modified Strip Method Utilisation for Wheel/rail Contact Stress Evaluation. $9^{\text {th }}$ intern. Conference on contact mechanics and wear of rail/wheel system (CM2012): Southwest Jiaontong University, 2012. Presented in Chengdu, 2012.

[5] GERLICI, J., LACK, T.: Contact Geometry Influence on the Rail/Wheel Surface Stress Distribution, Procedia Engineering, vol. 2, No. 1, 2010, 2249-2257.

[6] LACK, T., GERLICI, J.: Wheel/rail Tangential Contact Stress Evaluation by Means of the Modified Strip Method. Communications - Scientific Letters of the University of Zilina, vol. 16. No. 3a, 2014, 33-39. ISSN 1335-4205.

[7] LACK, T., GERLICI, J.: Wheel/rail Contact Stress Evaluation by Means of the Modified Strip Method. Communications - Scientific Letters of the University of Zilina, vol. 15. No. 3, 2013, 126-132. ISSN 1335-4205.

[8] LACK, T., GERLICI, J.: Tangential Stresses for Non-elliptical Contact Patch Computation by Means of Modified FASTSIM Method. Inter. Association of Vehicles system Dynamics (IAVSD) 2013: 23 ${ }^{\text {rd }}$ intern. symposium on dynamics of vehicles on roads and tracks, August 2013, Qingdao : Chengdu : Southwest Jiaotong University, 2013. USB key, [6] p. 


\section{COMMNICOIIONS}

[9] FEHR, J., EBERHARD, P.: Simulation Process of Flexible Multibody Systems with Non-modal Model Order Reduction Techniques. Multibody System Dynamics (2011) 25: 313-334, Springer Science + Business Media B.V, 2010. DOI 10.1007/s11044-010-9238-3.

[10] SHABANA, A. A.: Flexible Multibody Dynamics: Review of Past Recent Developments. Multibody System Dynamics, 1: 189-222, 1997.

[11] NOWAKOWSKI, Ch., FEHR, J., FISCHER, M., EBERHARD, P.: Model Order Reduction in Elastic Multibody Systems using the Floating Frame of Reference Formulation. Institute of Engineering and Computational Mechanics: University of Stuttgart: Germany.

[12] VASKO, M., LEITNER, B., SAGA, M.: Computational Fatigue Damage Prediction of the Lorry Frames under Random Excitation, Communications - Scientific Letters of the University of Zilina, vol. 12, No. 4, 2010, $62-67$.

[13] LEITNER, B.: Fatigue Damage Analysis and Fatigue Life Prediction of Lorry Frame under Random Excitation. Transport means 2014. Proc. of the $18^{\text {th }}$ intern. conference, October 2014, Kaunas University of Technology, 2014, 9-14, ISSN 1822-296X.

[14] STASTNIAK, P., HARUSINEC, J., LACK, T., GERLICI, J.: Structural Design of Freight Railway Wagon. Proc. of conference Innovations in the conception, design, manufacture and testing of freight wagons I, 27-135, November 2014, Zilina: University of Zilina, ISBN 978-80-554-0955-9.

[15] STASTNIAK, P., HARUSINEC, J.: Computer Aided Simulation Analysis for Computation of Modal analysis of the Freight Wagon. Communications - Scientific Letters of the University of Zilina, vol. 15. 2013, 73-79. ISSN 1335-4205.

[16] SKOCILAS, J., SKOCILASOVA, B., SOUKUP, J.: Determination of the Rheological Properties of Thin Plate under Transient Vibration. Latin American J. of Solids and Structures. Brazil Society for mechatronics and Engineering. ISSN 1679-7817 (print), 1679-7825 (online)

[17] SOUKUP, J., VALES, F., VOLEK, J., SKOCILAS, J.: Transient Vibration of Thin Viscoelastic Orthotropic Plates. Acta Mechanica Sinica, vol. 27, No. 1, pp. 98-107. The Chinese Society of Theoretical and Applied Mechanics; Institute of Mechanics, Chinese Academy of Sciences, co-published with Springer, ISSN 0567-7718 (Print, 1614-3116 (online).

[18] SIMPACK documentation 2014, user guide (part of the program package).

[19] DIZO, J.: Simulation Analysis of a Freight Wagon Running on a Real Track. Proc. of Innovations in the conception, design, manufacture and testing of freight wagons I, November, 2014, Zilina: University of Zilina, 2014, ISBN 978-80-554-0955-9, pp. 127. 135 .

[20] DIZO, J., GERLICI, J., LACK, T.: The Goods Wagon Equipped by Y25 Bogies Computer Simulation Analysis. Transcom 2013, Proc. of the $10^{\text {th }}$ European Conference of Young Researchers and Scientists, University of Zilina, ISBN 978-80-554-0695-4. - pp. 63-66.

[21] HARUSINEC, J., DIZO, J., STASTNIAK, P.: The Computer Simulation of the Goods Wagon by Y25 Bogies (in Slovak). Technolog, pp. 245-250, 2013, University of Zilina, ISBN 1337-8996.

[22] STASTNIAK, P., HARUSINEC, J., GERLICI, J., LACK, T: Structural Analysis of the Construction Freight Bogie Wagon (in Slovak). Strojirenska technologie - The J. for Science, Research and Production, vol. 18, No. 2, 2013, 105-11. ISSN 1211-4162. 